\title{
Design of Unmanned Monitoring and Automatic Monitoring System
}

\author{
Xudong Ding, Hongyan Jiang, Weihua Zhou
}

School of Mechanical Engineering and Automation, Nanjing Institute of Mechanical and Electrical Engineering,

Chongqing, China

\section{ABSTRACT}

With the development of electronic technology, medical equipment is also undergoing some changes, and the traditional manual monitoring mode is clearly unsatisfied with today's monitoring needs. At present, China's medical institutions in the infusion treatment, the infusion rate control or the use of conventional clinical infusion, general use of hanging bottle infusion, and direct observation with the eyes, relying on manual clip to control the infusion rate, this mode of operation cannot be precisely controlled Infusion speed, and the workload. Therefore, the need for both cheap and practical medical infusion detection monitoring device to meet today's medical needs. The system is designed with the single chip AT89C51 as the core, the keyboard and photoelectric sensor as the input system to digital tube and motor as the output system of intelligent infusion control and monitoring system. The keyboard system is a stand-alone key system, the function of the photoelectric sensor for the liquid droplet speed and infusion bottle page height of the reliable detection. When the liquid level is below the alert value, the system issues an alarm and can manually remove the alarm. The motor has a large controllable power and the input pulse can be maintained when the same torque and other advantages, so that you can control the bottle by the upper and lower slowly to achieve the purpose of intelligent control of liquid drip speed.

Keywords: single-chip; photoelectric sensor; stepper motor

Received: $18^{\text {th }}$ Jan. 2019 Accepted: $6^{\text {th }}$ Apr. 2019 Online: $20^{\text {th }}$ Apr. 2019

\section{Introduction}

\subsection{Research background and significance}

With the rapid development and application of microelectronics technology and information technology, there is a quiet revolution in information technology in the field of medical equipment. Especially in recent years, the highly developed modern technology is for the liquid drip automatic monitoring provides a solid and reliable technical foundation. China is the most populous country in the world. With the rapid development of various fields in recent years and the deterioration of the environment, the increasing number of diseases and the increasing consumption of medical consumers. Although the reform and innovation of health care government every year into a huge financial support, but the effect is still minimal, and western developed countries, there is a certain gap between the technology. At present, china's medical equipment market share in the global share is still very small. According to the requirements of the state economic and trade commission, china in 2050 or so to become the world's medical equipment manufacturing power. Thus, China's medical technology reform and innovation has great prospects.

Intravenous infusion in the medical work is widely used, according to statistics hospitalized infusion rate of $70-80 \%$ [1], it is not only an important route of administration, but also to patients with body fluids, nutrition, an important way the drip infusion is a common clinical use of treatment, has long been relying on manual control. In the traditional infusion [2], prone to some unusual circumstances, such as pipeline blockage, drip rate abnormalities and infusion after the completion of such conditions. If these abnormal circumstances cannot be found in time, it will cause harm to the patient, serious and even cause medical malpractice. Therefore, the design of an unmanned monitoring system of medical equipment to improve the quality of our medical treatment has a very important help, such as (A-A) imagination.

Copyright (C) 2019 Ding et al. This is an Open Access article distributed under the terms of the Creative Commons Attribution - NonCommercial 4.0 International License (http://creativecommons.org/licenses/by-nc/4.0/), permitting all non-commercial use, distribution, and reproduction in any medium, provided the original work is properly cited. 


\subsection{Development and research status at home and abroad}

In order to improve the safety of the infusion process and reduce the work intensity of the medical staff during the infusion process, the intelligent monitoring of the infusion process has been studied and tested at home and abroad. Drip infusion monitoring equipment development has experienced the following stages:

(1) Mechanical type

The method appears earlier, the use of liquid infusion bottle in the liquid level will lead to the decline in the principle of weight, with a spring scale to detect the liquid level. Due to the different weight of the infusion bottle, the actual situation is more complicated, this method is less reliable, the operation is inconvenient and the applicability is poor.

(2) Capacitive type

When the infusion bottle in the liquid level changes, causing changes in the length of the liquid to monitor the liquid level. Such monitoring equipment reliability, practicality is relatively high, but because the size of the infusion bottle shape is not a unified standard, because the infusion monitoring equipment requires a variety of design specifications, in use will not put, cannot put into practical use.

(3) Electrode type

The positive and negative electrodes are inserted into the murphy tube of the infusion set, and when the liquid drops down, an interrupt signal is generated, thereby monitoring the speed of the drip. This type of equipment is reliable, simple to design, but in the process of drop will be in contact with the electrode, may cause some medical and health problems.

(4) Photoelectric type

Photoelectricity is the more popular drip monitoring in recent years. It is the use of liquid absorption and scattering way to weaken the intensity of infrared light through the liquid to measure. The transmitter and the receiver are installed on both sides of the moffeitube. When the liquid drips through the two, the sensor senses the change of the light intensity, converts the optical signal into an electrical signal, and is processed by the next processing unit to carry out the conversion process, thus monitoring the infusion process due to the photoelectric sensor and the liquid is not in contact, but also with the liquid, infusion equipment has nothing to do, so that the performance of infusion monitoring equipment has greatly improved, with easy to use, high efficiency and applicability of the advantages [3].

\section{System Solutions}

\subsection{System basic configuration}

\subsubsection{Liquid drip speed detection}

The use of light reflection principle. Using reflective infrared photoelectric sensor detection. Infrared light emitting diode perpendicular to the funnel wall to send infrared light, infrared receiver tube according to the strength of the received infrared light signal to generate pulse signal, first through the amplifier circuit processing and then through the voltage comparison circuit can be processed by the microcontroller can handle the pulse signal the implementation of the block diagram shown in Figure 1. Wherein the shaping circuit includes an amplifier circuit and a comparison circuit. This scheme utilizes the principle of light reflection. Using reflective infrared photoelectric sensor detection. Directly reflective photoelectric switch is a set of transmitters and receivers in one of the sensors, when there is a detected object through the photoelectric switch transmitter to send a sufficient amount of light reflected to the receiver, so the photoelectric switch to produce a switch signal. When the surface of the object to be detected or its high reflectivity, the direct reflection of the photoelectric switch is the preferred detection mode. 


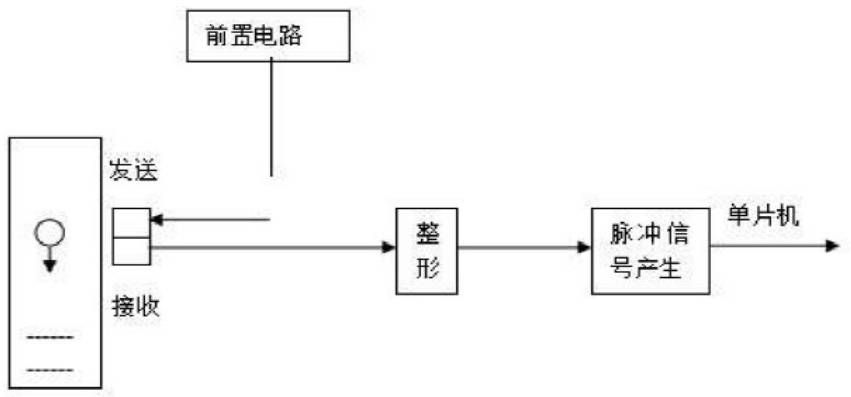

Figure 1. Light reflection principle detection circuit

\subsubsection{Liquid level inspection of infusion bottles}

Option 3: The software can be set by detecting the drip speed to generate the alarm signal, so you can remove the liquid level detection circuit and completely by the liquid droplet speed detection circuit instead. This eliminates the need for a hardware reservoir liquid level detection circuit, which is controlled by software.

\subsubsection{Keyboard scheme}

Using a stand-alone key circuit, each button occupies an I / O interface line, each I / O port working state does not affect each other, such as a port using port direct scan mode. The disadvantage is that when the keys are more occupied by the single-chip I / O port more, the advantages of simple circuit design, and programming is extremely easy.

\subsubsection{Display the program}

Using three LED seven digital display shows the number of drops. Digital tube with low energy consumption, low loss, low pressure, long life, anti-aging, lower requirements on the outside world. At the same time digital tube using BCD encoding display numbers, the program is easy to compile, less resource consumption.

\subsubsection{Motor system solutions}

The motor plus output direct feedback control system was shown in Figure 2. The implementation of the program is the motor, the motor is used to adjust the height of the storage bottle, while changing the height of the drop bucket, so as to achieve the purpose of changing water droplets. Use the photoelectric sensor to test the speed of water droplets, and then compare the amount of feedback to the controller, and then adjust the height then compare the amount of feedback. Until the transfer to the desired water droplet speed, this system is a direct feedback output of the closed-loop automatic control system. In the control of the stability of speaking, can be more stable regulation of water droplets speed, and then because the output directly as a feedback, is conducive to the stability of the output.

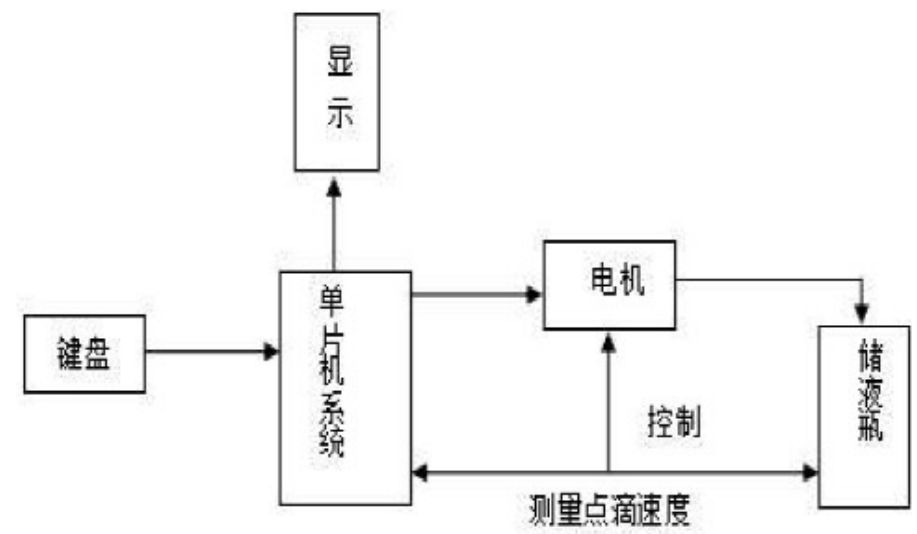

Figure 2. Motor drive circuit diagram

On the one hand to adjust the steps as small as possible, positioning is better; the other hand, if the stop signal arrival, to be able to immediately stop the motor. So choose stepper motor, this program is most suitable. 


\subsubsection{Drip speed control program}

Using single-chip and programmable logic devices to control the tightening of the infusion hose to control the drip speed. Change the shape of the plastic dropper to control the flow rate of the liquid. Although this method is intuitive, but there are many shortcomings. First, due to the pressure on the wall to change its shape, the pressure applied to the relationship between the flow of non-linear, which brings difficulty to the flow control. Second, because the dropper is made of plastic, after a long time after the release of pressure and cannot make the plastic drip tube completely restored prototype, the control device cannot guarantee the desired control effect. In addition, to complete the production of drip folder has some difficulties. Even if this program has many shortcomings, but its structure is small, strong mobility, low motor requirements, the advantages of simple mechanical equipment [6].

\subsection{Basic system block diagram}

According to the requirements of the system it can be divided into the following modules: drip speed measurement module, liquid level detection module, keyboard display module, motor system control (drip speed control) module. The basic system block diagram [7] as shown in Figure 3 .

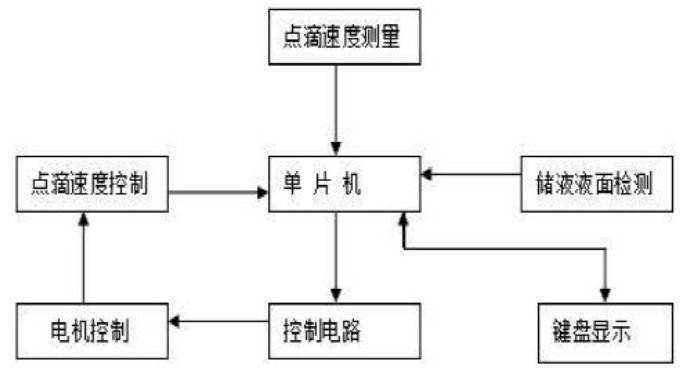

Figure 3. System block diagram

\section{System Hardware Design and Implementation}

\subsection{The basic components of the system hardware}

The use of detection technology, automatic control technology and electronic technology. The system can be divided into a sensor detection section and a control section.

Sensor detection part: the system uses the infrared tube to send and receive the photoelectric sensor will detect the signal into the controller can identify the electrical signal. The sensor detection circuit includes two-unit circuits: a drip rate measuring circuit and a liquid storage detection circuit.

Control part: the control device in the system according to the sensor to transform the output of the electrical signal to determine the logic to control the speed of the drip and digital display, to complete the drip device automatic detection, automatic speed control, digital display and alarm functions and other tasks the control part of the main include three circuits: single-chip control circuit, the motor drive circuit, digital tube dynamic display circuit.

\subsection{Liquid drip rate detection circuit}

Liquid drip velocity detection is an important part of the whole system. Detection accuracy is one of the most important indicators to measure the accuracy of the system. The dropper is placed in the middle of the grooved optocoupler. During the detection process, the droplet moves in a nearly elliptical downward direction and passes through the grooved optocoupler. As the surface of the droplet is curved, the upper and lower half of the light will be refracted twice, so that the receiver cannot receive light, only in the middle of the droplets of light can be directly through, resulting in such a waveform is unstable, interference large, the system uses a timer NE555 connected to a monostable trigger, the output pulse waveform shaping and then enter the microcontroller. Because the system drip rate range of 60-80 drops / min, with the fastest request to count, 80 points per minute, the minimum interval between two drops of $0.75 \mathrm{~s}$, the design of the monostable trigger should make temporary state of time less than $0.75 \mathrm{~s} . \mathrm{R}=400 \mathrm{~K}, \mathrm{C}=1 \mathrm{uF}$, then the temporary steady 
state time $\mathrm{T}=1.1 \mathrm{RC}=0.44 \mathrm{~s}$, can effectively avoid the interference between the two droplets. When there is no droplet dripping, the maximum light intensity through the sensor, the maximum photocurrent generated, making the photosensitive transistor conduction, and its collector and emitter voltage is very small, the output low; when there are droplets down, cut off the optical path, resulting in reduced photocurrent, phototransistor medium, the output high-level, according to the output pulse level can detect whether the droplets go through [8].

\subsection{Liquid storage tank liquid level detection circuit}

Level detection refers to the infusion, when the liquid level below the given infusion position, that the infusion process has ended, should be issued an alarm signal to stop the infusion operation to ensure that the patients' safety level detection circuit schematic diagram shown in Figure 4 and 5 . When the liquid level inside the bottle is above the optical path, the infrared receiving transistor cannot receive the light from the infrared emission diode, so that the positive input of the amplifier is ' 1 '; when the liquid level in the bottle gradually drops to the vicinity of the optical path, The infrared receiving diode can receive the infrared emission diode to send the light, thus turning on, the amplifier positive input is ' 0 ', then drive the corresponding circuit to send an alarm signal to inform the health care workers to take timely measures. The remaining amount of infusion when the alarm should be sufficient to meet the nursing staff on the remaining amount of liquid [9].

The alarm signal is also determined by the liquid storage signal. When the liquid level of the liquid level is $2-3 \mathrm{~cm}$, it will be generated by the infrared tube to send and receive detection signal, that is, the alarm signal. The alarm circuit is shown in Figure 6 [10].

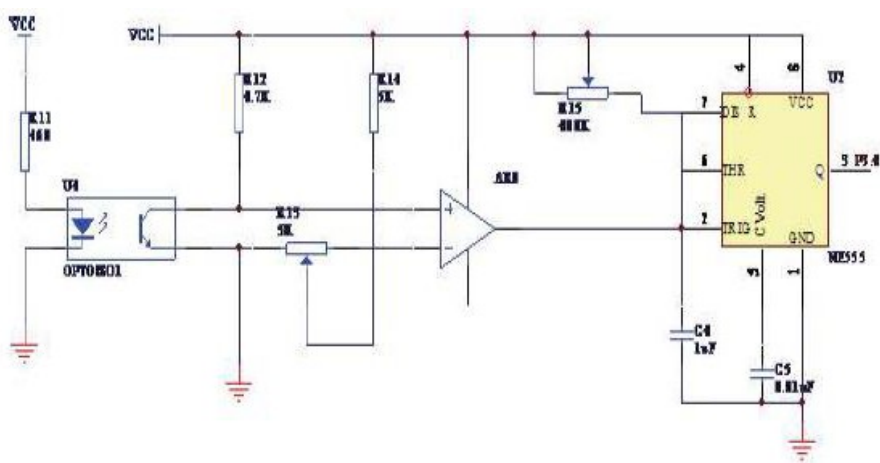

Figure 4. Liquid drip rate detection circuit

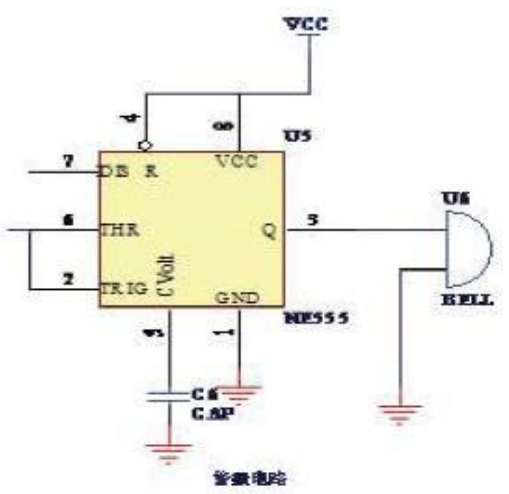

Figure 6. Alarm circuit

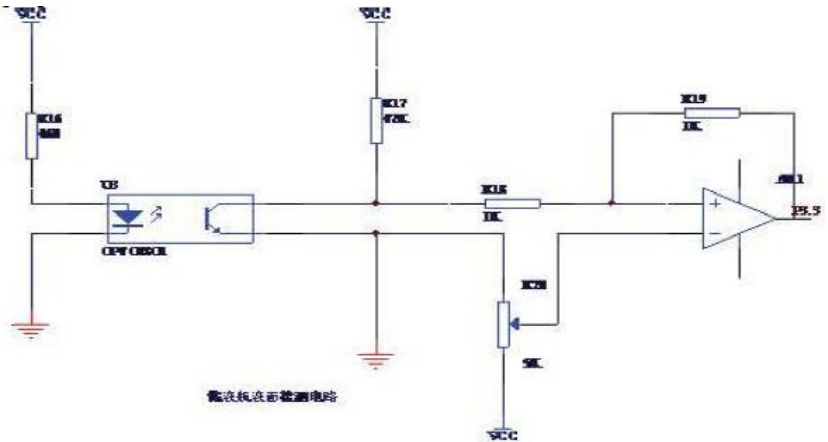

Figure 5. Liquid level detection circuit for liquid storage bottles 


\subsection{Keyboard circuit}

Each button switch is connected to the $10 \mathrm{~K}$ of the next resistance, so that it is in the off state of the output bit low, rather than floating state. To set the first to press the 'Ctrl' button, the output high to the microcontroller $\mathrm{P} 0.0$, that set the start. Then press the button ' ++ ', the output high to the microcontroller P0.1; If you press the button '-', the output high to the microcontroller P0.2. Exit set, require both to P0.1 and P0.2 to high, that is, need to press the button ' ++ ' ' - ' on the microcontroller to explain. The keyboard circuit is shown in Figure 7.

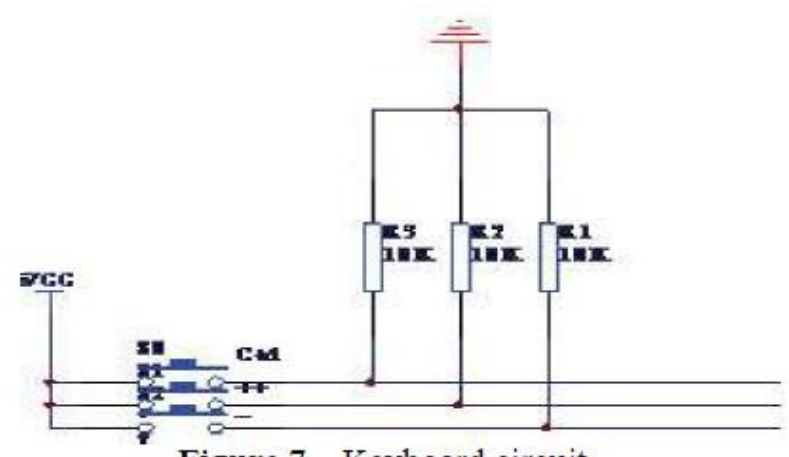

Figure 7. Keyboard circuit

\subsection{Display circuit}

Using dynamic display mode to drive three seven-segment digital tube, to show the speed of bit. Digital tube with a total cathode.

\subsection{Stepper motor drive circuit}

The motor drive circuit is shown in Figure 8. The road uses the H-type switch drive circuit. The entire circuit can be divided into two levels: the first level of the microcontroller I / O port, with the emitter follow the circuit to increase the current drive capacity; the second level is the drive circuit. When P3.0 is high, Q2 is turned on, Q1, Q3 conduction, the motor voltage at both ends of $+5 \mathrm{~V}$, the motor is running; when P3.1 is high, Q5 conduction, Q4, Q6 conduction, the voltage across the motor is $-5 \mathrm{~V}$ and the motor is reversed.

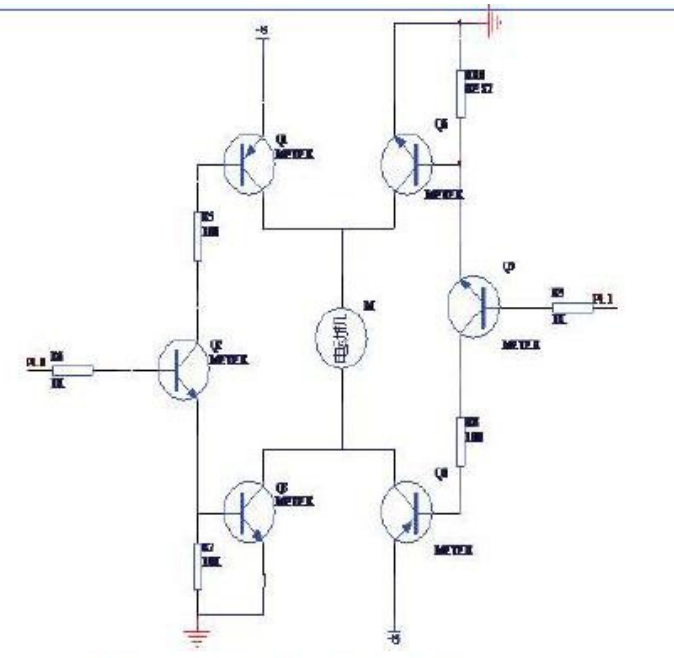

Figure 8. Motor drive circuit

\section{System Software Design}

The main program initializes the module, receives the signal from the sensor, and processes the signal, the output adjustment signal controls the rotation of the motor, and the scanning keyboard is dynamically displayed. Using the circular query mode, to display and control the speed of bit. 


\subsection{Liquid drip rate detection subroutine}

Detection of drip rate subroutine is mainly used with the system signal acquisition. After the photoelectric sensor acquisition, after the Schmitt trigger shaping, the output to the microcontroller port into the microcontroller internal. The sub-program for detecting the drip rate is shown in Figure 9.

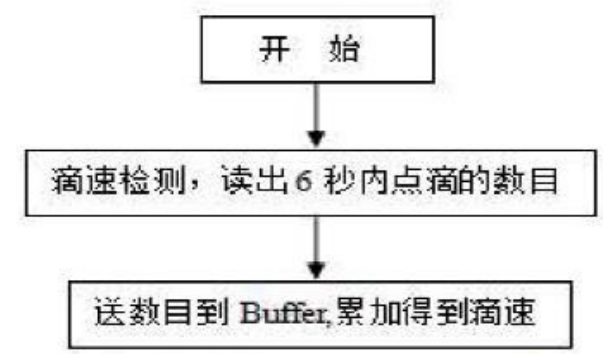

Figure 9. Liquid drip rate detection subroutine

\subsection{Liquid level detection sub-program}

This procedure is mainly used to measure the liquid level inside the liquid level, when the liquid level is lower than 2$3 \mathrm{~cm}$, the alarm signal. If the height of the liquid level is not in this interval, the drip rate is usually collected and sent to the inside of the microcontroller. The flow chart of the storage subroutine is shown in Figure 10.

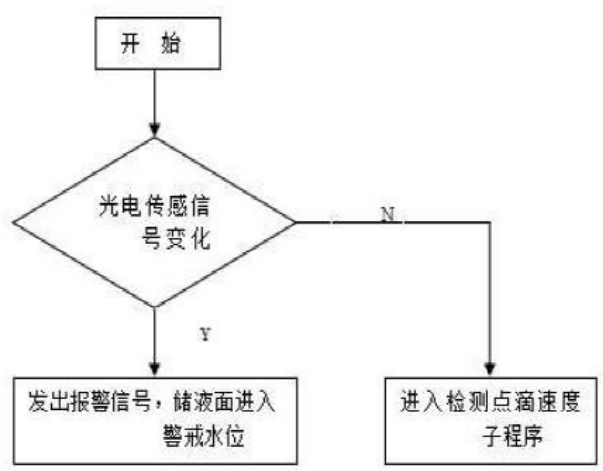

Figure 10. Liquid level detection subroutine

\subsection{Bit rate detection subroutine}

According to the design requirements of the subject, first read the number of bits within 6 seconds (will be divided into a minute average), and then get the current bit by bit speed. According to its set speed value and the current bit rate of the error to determine the motor forward and reverse, that is, control the location of the storage bottle level, and further affect the next 6 seconds of the bit rate, such a cycle reciprocating. The drip speed of the motor control program flow chart shown in Figure 11.

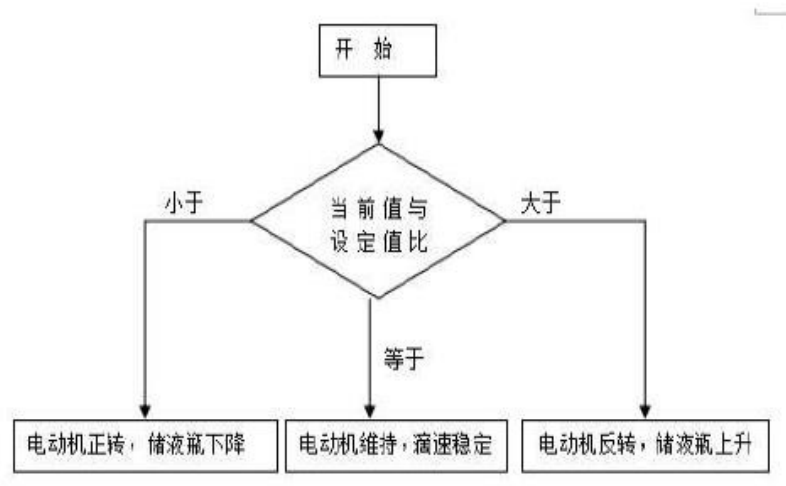

Figure 11. Drip rate detection subroutine 


\subsection{Keyboard display subroutine}

Keyboard and display word program are mainly used to determine whether the speed has to adjust the movement and drip speed display (including the current value and set value). The program flow chart shown in Figure 12

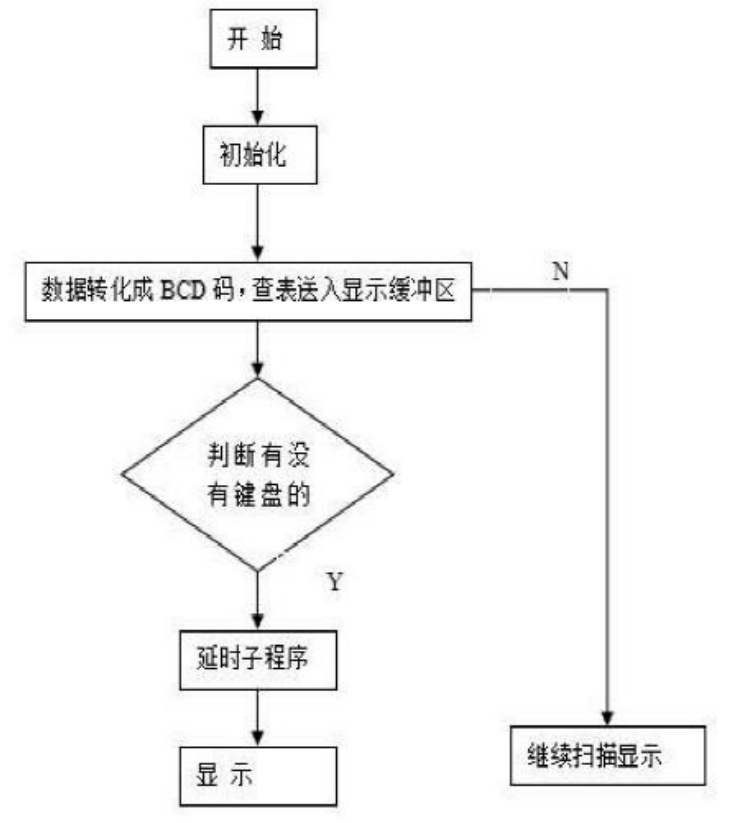

Figure 12. Keyboard display subroutine

\subsection{System of the main program design}

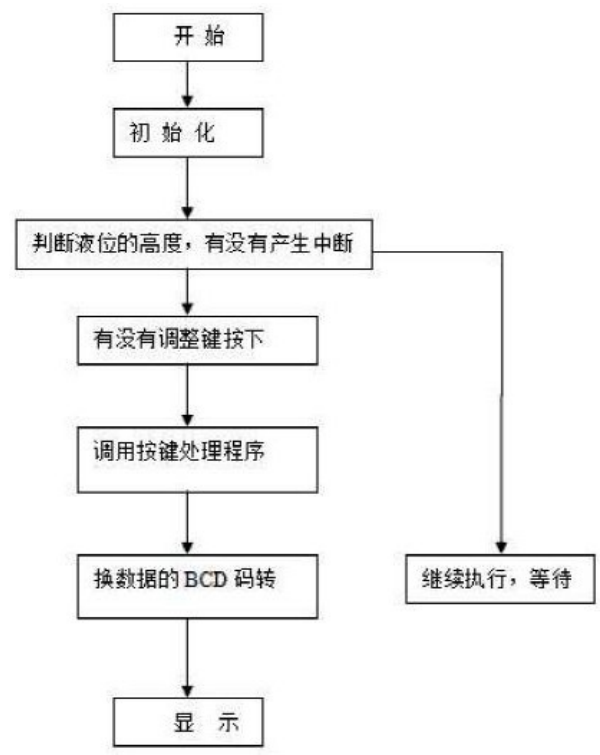

Figure 13. System main program flow chart

\section{References}

1. ZT. Wang, RF. Wang, TF. Yan. Intelligent liquid drip speed monitor. Automation and Instrumentation, 2004, (5): 48-50

2. DT. Zhang, W. Wei. Intelligent medical drip control system design and implementation. Jilin Journal of Engineering and Technology, 2009,25 (10)

3. XL. Song, HL. Wang, JL. Wan. Design of liquid droplet speed monitoring device based on single chip microcomputer system. Computer development and application, 2007, 20 (5). 
4. LP. Zhu. Single-chip microcomputer control double stepper motor motion control system. Electronic Communication and automatic control technology, 2008,8.

5. Design and Development of General Infusion Alarm and Control System [J]. Medical Equipment Information, 2004 (9): 10-11 (in Chinese with English abstract)

6. W. Xiao, YQ. Tu, J. Wang, etc. Liquid droplet wireless intelligent monitoring system design and implementation [J]. Electronic Measurement Technology, 2008 (1): 133-136

7. M. Yin, Y.Xie. Design of a new wireless infusion monitoring system [J]. Computer lu0026 Digital Engineering, 2007 (8): 176-178 